Anesthesiology Clin 34 (2016) 511-523

\title{
Multimodality Neuromonitoring
}

\section{Matthew A. Kirkman and Martin Smith*}

Neurocritical Care Unit, The National Hospital for Neurology and Neurosurgery, University College London Hospitals, Queen Square, London, UK.

* Corresponding author email: martin.smith@uclh.nhs.uk

Word Count: $\quad 5486$

Tables: $\quad 2$

Figures: $\quad 0$

Disclosure statement: MS is part funded by the Department of Health National Institute for Health Research Centres funding scheme via the University College London Hospitals/University College London Biomedical Research Centre.

Conflicts of interest: None.

Key words: autoregulation; cerebral perfusion pressure; cerebrovascular reactivity; informatics; intracranial pressure; microdialysis; multimodality neuromonitoring. 


\section{Key points}

- The clinical neurologic examination is the cornerstone of neuromonitoring, but a complete clinical assessment is not possible in intubated and sedated/anesthetized patients.

- There are several techniques that permit global or regional monitoring of cerebral hemodynamics, oxygenation, metabolism and electrophysiology, invasively or non-invasively.

- Given the pathophysiological complexity of acute brain injury, a single neuromonitor is unable to detect all instances of cerebral compromise.

- Multimodality neuromonitoring is widely used to individualize patient management after acute brain injury.

- High-quality outcome studies are necessary to demonstrate any outcome effects of multimodal neuromonitoring-guided treatment.

\section{Article synopsis}

The monitoring of systemic and central nervous system physiology is central to the management of patients with neurologic disease in both the perioperative and critical care settings. There exists a range of invasive and non-invasive, global and regional monitors of cerebral hemodynamics, oxygenation, metabolism and electrophysiology that can be used to guide treatment decisions after acute brain injury. With mounting evidence that a single neuromonitor cannot comprehensively detect all instances of cerebral compromise, multimodal neuromonitoring allows the adoption of an individualized approach to patient management based on monitored physiologic variables rather than a generic 'one size fits all' approach targeting pre-determined and often empirical thresholds. The evidence base for outcome benefits of multimodal 
neuromonitoring-guided treatment is currently poor, and it remains unclear how a derangement in one physiological variable in the presence of normal values in others should be managed. Crucial to the widespread implementation of multimodality neuromonitoring is the development of high-quality outcomes studies to determine whether adoption of a multimodal neuromonitoring approach improves outcomes, and which modalities are more useful than others in guiding treatment of the acutely injured brain. 


\section{INTRODUCTION}

Systemic and central nervous system physiological monitoring is used to guide the management of patients with neurologic disease in the perioperative and critical care settings. Whilst the clinical neurologic examination is the cornerstone of neuromonitoring, a complete clinical assessment is not possible in intubated or sedated/anesthetized patients. Several techniques are available for global or regional monitoring of cerebral hemodynamics, oxygenation, metabolism and electrophysiology to guide patient management.

The pathophysiology of acute brain injury (ABI) is complex, involving changes in cerebral blood flow (CBF), oxygen and glucose delivery and utilization, and electrophysiological derangements. A single monitoring modality is therefore unable to detect all instances of cerebral compromise. Multimodality neuromonitoring is the simultaneous measurement of several variables simultaneously, and provides a more comprehensive picture of the (patho) physiology of the injured brain and its response to treatment. It allows an individually tailored approach to the management of patients with $\mathrm{ABI}$ in which treatment decisions are guided by monitored changes in pathophysiological variables rather than generic 'one size fits all' treatment targets. General indications for neuromonitoring are shown in table 1.

Table 1 near here

This chapter will review the neuromonitoring techniques commonly used in perioperative and critical care settings. Some important modalities are covered elsewhere in this edition and will not be discussed here. The reader is referred to 
chapters 8 and 9 for detailed discussions of intraoperative neurophysiological monitoring and brain oxygenation monitoring respectively.

\section{INTRACRANIAL PRESSURE}

The monitoring and management of intracranial pressure (ICP) is the cornerstone of neuromonitoring in patients with traumatic brain injury (TBI), and increasingly used in other brain injury types. In addition to measuring absolute ICP, ICP monitoring permits the calculation of cerebral perfusion pressure (CPP), a therapeutic target in itself, identification and analysis of pathological ICP waveforms, and derivation of indices of cerebrovascular pressure reactivity.

\section{Intracranial monitoring methods}

ICP is most commonly measured using an intraventricular catheter or parenchymal microtransducer device [1]. Ventricular catheters measure the pressure of the CSF in the lateral ventricles, which is an assessment of global ICP. This can be achieved using a standard ventricular catheter connected via a fluid-filled system to an external pressure transducer, or a catheter incorporating microstrain gauge or fiberoptic technology. Both allow in-vivo calibration and therapeutic drainage of CSF [2]. Ventricular catheter insertion can be technically challenging, and associated with placement-related hemorrhage and catheter-associated ventriculitis. The risk of ventriculitis increases with time following catheter insertion, but can be reduced by the use of antibiotic-impregnated or silver-coated catheters.

Two broad categories of parenchymal microtransducer ICP monitoring systems exist. Solid-state piezoelectric strain gauge devices incorporate pressure-sensitive resistors 
which translate pressure generated changes in resistance to an ICP value. Fibre optic devices transmit light towards a mirror at the catheter tip which becomes distorted by changes in ICP. The difference in the intensity of reflected light as the mirror distorts are translated into ICP values. Compared to ventricular catheters, parenchymal ICP measurement devices are easy to insert and have a better safety profile, particularly with regards to hematoma and infection risk [1]. They are usually placed approximately $2 \mathrm{~cm}$ into brain parenchyma through a cranial access device or at craniotomy when they can also be sited subdurally. Intraparenchymal devices measure localized pressure, but provide equivalent pressure measurements to ventricular catheters in most circumstances. Zero drift, whereby there is a change in baseline ICP readings, is associated with microtransducer systems and can result in measurement error over several days. Furthermore, in-vivo recalibration is not possible with parenchymal devices.

Several non-invasive ICP monitoring techniques are available, but most have limitations [3]. A pulsatility index derived from transcranial Doppler (TCD) ultrasonography is an imprecise assessment of ICP compared to invasive measurement alternatives. Optic nerve sheath diameter (ONSD), measured by ultrasound or computed tomography (CT), is related to ICP and has been used to identify intracranial hypertension. All currently available non-invasive ICP monitoring techniques fail to measure ICP sufficiently accurately for routine clinical use, and most are unable to monitor intracranial dynamics continuously [4].

\section{Indications for intracranial pressure monitoring}


There are no high-quality data confirming an association between ICP-guided management and improved outcomes in any brain injury pathology. Nevertheless, ICP monitoring is a standard of care after severe TBI in most neuroscience centers [2]. Guidelines from the Brain Trauma Foundation (BTF) published in 2007 contain recommendations for the use of ICP monitoring [5], and a more recent expert statement provides updated guidance [6]. The key features of these recommendations are summarized in table 2.

Table 2 near here

Aside from TBI, ICP monitoring provides valuable information to guide the critical care management of aneurysmal subarachnoid hemorrhage (SAH) [7] and intracerebral hemorrhage (ICH) [8]. It is also standard in the management of hydrocephalus, including chronic monitoring of normal pressure hydrocephalus, and becoming so in the perioperative management of patients with neoplastic lesions and associated mass effect. However, these indications are not as well-defined or wellstudied as those for TBI.

\section{Intracranial pressure monitoring-guided treatment}

Normal mean ICP is $5-10 \mathrm{mmHg}$ in healthy, resting supine adults. It is generally advised that ICP greater than 20-25 mmHg requires treatment after TBI [5], but higher and lower thresholds have also been recommended [2]. It is well known that intracranial hypertension is detrimental to outcome, but crucially it is the time spent above a defined ICP threshold as well as absolute ICP values that are important determinants of poor outcome. Changes in the ICP waveform are observed as ICP 
rises, and waveform analysis has been utilized to predict the onset of intracranial hypertension [9]. Evidence that this translates into more timely intervention and improved outcomes is currently lacking.

A meta-analysis incorporating 14 studies of 24,792 patients with severe TBI found that ICP monitoring-guided management of intracranial hypertension was associated with no significant overall mortality benefit compared to treatment without ICP monitoring, although mortality was lower in those who underwent ICP monitoring in studies published after 2012 [10]. The only randomized controlled trial of ICP-guided management after TBI (Benchmark Evidence from South American Trials: Treatment of Intracranial Pressure, BEST:TRIP) found similar three- and six-month outcomes in patients in whom treatment was guided by ICP monitoring compared to treatment guided by imaging and clinical examination in the absence of ICP monitoring [11]. Those in the non-ICP monitored group received protocol-specified but empirical treatment on a fixed schedule basis, and the wider applicability of such an approach is questionable given that one of the interventions (mannitol) has been shown to have a more beneficial effect when directed by monitored rises in ICP. In contrast to previous studies [12], those undergoing ICP monitoring in the BEST:TRIP trial received significantly fewer days of ICP-directed treatment (hyperventilation, hypertonic saline/mannitol and barbiturates) compared to those in the non-ICP monitored group, although the length of intensive care unit (ICU) stay was similar. Whether the findings of this study, which was conducted in Bolivia and Ecuador, are applicable to populations with access to superior pre-hospital care and rehabilitation services remains to be seen. However, this study is important because it reinforces the principle that the evaluation and diagnosis of intracranial hypertension, whether by 
monitoring ICP or assessment of clinical and imaging variables, is central to the management of patients with severe TBI.

ICP monitoring cannot, and is not designed to, assess the adequacy of cerebral perfusion. Several studies confirm that brain hypoxia/ischemia can occur when ICP and CPP are within established thresholds for normality [13]. Moreover, elevated ICP values can arise from both increased cerebral blood flow (hyperemia) and reduced cerebral blood flow secondary to cerebral edema, highlighting the non-specific nature of ICP readings. There is also evidence that multimodality monitoring incorporating brain tissue partial pressure of oxygen $\left(\mathrm{PtiO}_{2}\right)$ monitoring in addition to ICP can identify cerebral hypoperfusion more reliably than ICP monitoring alone [14]. ICP monitoring is therefore best considered as one part of a multimodal neuromonitoring strategy rather than as a monitoring modality in isolation.

\section{CEREBRAL PERFUSION PRESSURE}

Cerebral perfusion pressure is calculated as the difference between mean arterial pressure (MAP) and ICP. Accurate calculation of CPP requires that the zero reference points for both MAP and ICP should be the same, i.e. at the level of the brain using the tragus of the ear as the external landmark [15]. This is crucial if the head of the bed is elevated, as is routine in the management of ABI. Under such circumstances, measuring arterial blood pressure (ABP) at the level of the heart and ICP at the level of the brain results in an erroneously high calculated CPP; a measured CPP of 60 mmHg may actually represent a 'true' CPP of $<45 \mathrm{mmHg}$ [15]. Such measurement discrepancies are exacerbated in tall patients, with varying elevations of the head of the bed, and different sites of arterial cannulation. Although international management 
guidelines recommend target values for CPP, the calibration of blood pressure, which directly influences calculated CPP values, is not described. A recent narrative review was unable to determine how MAP was measured in the calculation of CPP in 50\% of 32 widely cited studies of CPP-guided management [16]. There have been recent calls for the adoption of international standardization of CPP measurement methods, not only in clinical practice but also in clinical trials [17].

\section{Cerebral perfusion pressure-guided treatment}

The indications for CPP monitoring are similar to those for ICP, with a predominant application in TBI [15] and emerging indications in other brain injury types [2].

The recommendations relating to CPP thresholds after TBI have changed over time. The most recent guidelines from the $\mathrm{BTF}$ recommend that $\mathrm{CPP}$ be maintained between 50-70 mmHg after TBI, with evidence of adverse outcomes with lower or higher values [5]. CPP that is too low risks cerebral hypoperfusion and ischemia, whereas targeting a higher CPP does not guarantee a favorable outcome and is associated with a substantial risk of acute lung injury related to the administration of large fluid volumes and inotropes/vasopressors to increase MAP [15]. Multimodality monitoring incorporating $\mathrm{PtiO}_{2}$ monitoring and autoregulatory indices has been used to identify an 'optimal' $\mathrm{CPP}$ value $\left(\mathrm{CPP}_{\text {opt }}\right)$ in an individual patient. Targeting $\mathrm{CPP}_{\text {opt }}$ rather than a generic CPP threshold minimizes the risks of excessive CPP and associated complications on the one hand, and cerebral hypoperfusion and worsening secondary brain injury on the other [2].

\section{CEREBROVASCULAR REACTIVITY}


Cerebrovascular reactivity is a key component of cerebral autoregulation (CA) which can be disturbed or abolished by intracranial pathology as well as by some anesthetic and sedative agents. This may result in uncoupling of regional $\mathrm{CBF}$ and metabolic demand, and increase the risk of secondary cerebral ischemia. Standard methods of testing static and dynamic CA are interventional and intermittent with limited applicability in anesthetized or critically ill patients. Methods for the continuous monitoring of cerebrovascular reactivity at the bedside have recently been described.

\section{Pressure reactivity index}

The pressure reactivity of cerebral vessels determines the ICP response to changes in $\mathrm{ABP}$, with disturbed reactivity implying disturbed pressure autoregulation. In the normal brain, increases in ABP result in cerebral vasoconstriction within 5-15 seconds with an associated reduction in cerebral blood volume (CBV) and ICP. If cerebrovascular reactivity is impaired, CBV and ICP increase passively with ABP, with opposite changes when $\mathrm{ABP}$ is reduced. The pressure reactivity index (PRx), calculated as the moving correlation coefficient of consecutive time averaged data points of ICP and ABP over a 4-minute period, can be measured continuously as a marker of autoregulatory status [18]. An inverse correlation between ABP and ICP, indicated by a negative value for PRx, represents normal cerebrovascular reactivity, whereas an increasingly positive PRx defines a continuum of increasingly nonreactive cerebrovascular circulation when changes in ABP and ICP are in phase. After ABI, cerebral vasoreactivity varies with perfusion pressure and optimizes within a narrow range of $\mathrm{CPP}\left(\mathrm{CPP}_{\mathrm{opt}}\right)$ specific to an individual patient. Targeting PRx-defined $\mathrm{CPP}_{\mathrm{opt}}$ allows individualized management of $\mathrm{ABP}$ and ICP, and minimizes the risks 
of excessively high or low CPP that can be associated with reliance on a generic CPP threshold [19].

\section{Other autoregulatory indices}

Cerebrovascular reactivity can alternatively be assessed with an oxygen reactivity index (ORx), defined as the moving correlation between $\mathrm{PtiO}_{2}$ and CPP. The correlation between ABP and TCD-derived cerebral blood flow velocity (mean velocity index, Mx), and several near infrared spectroscopy (NIRS)-derived variables (e.g. cerebral oximetry index, COx) have also been described [20]. Recently, an innovative technique incorporating ultrasound-tagged NIRS for the measurement of microcirculatory CBF has been used to monitor CA continuously during cardiac surgery [21].

\section{Autoregulation-guided treatment}

The assessment of cerebrovascular reactivity, particularly using PRx, has become popular in some centres during the management of TBI and, more recently, after SAH and ICH. Abnormal PRx values, indicative of autoregulatory dysfunction, are associated with poor outcome after TBI and, in small studies, PRx-guided optimization of CPP has been associated with improved outcomes [19]. A recent systematic review confirmed that monitoring cerebrovascular reactivity, in addition to allowing optimization of CPP, is important in evaluating relationships between CBF, oxygen delivery and demand, and cellular metabolism after TBI [22].

PRx is considered a global measure of autoregulatory status, whereas ORx represents regional autoregulation because of the focal nature of $\mathrm{PbtO}_{2}$. Thus, findings of 
deranged ORx but normal PRx after ICH strongly suggests the presence of focal but not global autoregulatory failure [23].

NIRS-derived measures of cerebrovascular reactivity have also been used to guide brain protection protocols during cardiac surgery. The duration and magnitude of blood pressure below the lower limit of CA are independently associated with major morbidity and mortality after cardiac surgery according to NIRS-derived data [24].

\section{CEREBRAL BLOOD FLOW MONITORING}

Under normal physiological conditions cerebral pressure autoregulation maintains CBF constant over a wide range of CPP. CA is often impaired after $\mathrm{ABI}$, and $\mathrm{CBF}$ becomes increasingly pressure dependant as autoregulatory failure worsens. Monitoring CBF can therefore provide information not only about absolute or relative blood flow, but also autoregulatory status.

Kety and Schmidt described the first practical method for measuring CBF in 1945. Their method incorporated the Fick principle, and forms the basis of many current CBF measurement techniques. Two bedside methods for the continuous assessment of CBF are available.

\section{Transcranial Doppler ultrasonography}

TCD is an established, non-invasive technique for the real-time assessment of cerebral hemodynamics. Ultrasound waves are used to measure blood flow velocity (FV) through large cerebral vessels from the Doppler shift resulting from red blood cells moving through the field of view. The FV waveform resembles an arterial pressure 
pulse wave, and waveform analysis permits quantification of peak systolic, end diastolic and mean FVs. The pulsatility index, which provides an assessment of distal cerebrovascular resistance, can also be measured. The main disadvantages of TCD are its measurement of relative changes as opposed to absolute $\mathrm{CBF}$, and operator dependency. Long-term recordings are limited by the need for accurate and immovable probe fixation, and TCD is therefore best considered an intermittent monitoring technique.

\section{Indications}

TCD has several perioperative indications but is most widely used to monitor changes in cerebral perfusion during carotid endarterectomy (CEA). There is a good correlation between TCD-monitored variables and EEG changes of ischemia, and this has been used to guide the need for shunt placement [25]. TCD can also detect emboli as characteristic short-duration, high intensity 'chirps,' and waveform analysis allows differentiation between air and particulate emboli [26].

TCD is also used in the intensive care management of SAH where regular assessments assist in both the diagnosis and management of cerebral vasospasm. Middle cerebral artery (MCA) FV > $120-140 \mathrm{~cm} / \mathrm{s}$, or FV increases $>50 \mathrm{~cm} / \mathrm{s} /$ day from baseline, are indicative of developing or established cerebral vasospasm-related delayed cerebral ischemia (DCI). Mean MCA FV thresholds of $100 \mathrm{~cm} / \mathrm{s}$ and 160 $\mathrm{cm} / \mathrm{s}$ have been identified as the most accurate thresholds for the detection of angiographic and clinical vasospasm respectively [27]. Changes in CBF influence FV and the Lindegaard ratio, which compares ipsilateral MCA and extracranial internal carotid artery FVs, is often preferred to measurement of FV in a basal cerebral vessel 
in isolation [28]. Vasospasm is diagnosed by a Lindegaard ratio index $>3$, and severe spasm by a ratio $>6$.

Although TCD can detect severe vasospasm with a sensitivity of 97\% [29], it is an operator-dependent tool requiring skilled personnel for interpretation and can only assess a small number of large arteries. Further, in an analysis of 1,877 TCD examinations, almost $40 \%$ of patients with clinical evidence of DCI never had FVs that exceeded $120 \mathrm{~cm} / \mathrm{s}$ [30]. Such findings are likely to be related to inter-individual variability as well as causes of DCI other than vasospasm, highlighting that treatment decisions in the management of SAH-related DCI should not be based on TCD results alone.

TCD has been used to monitor the integrity of $\mathrm{CO}_{2}$ reactivity as well as pressure autoregulation after $\mathrm{ABI}$ and, as noted earlier, to provide a noninvasive but imprecise estimate of ICP. Clinical data on the use of TCD in brain injury types other than TBI and SAH are limited, and there have been concerns about accuracy and reliability [4].

\section{Thermal diffusion flowmetry}

Thermal diffusion flowmetry (TDF) is an invasive, continuous and quantitative monitor of regional CBF. The TDF catheter consists of a thermistor heated to a few degrees above tissue temperature and a second, more proximal, temperature probe. The temperature difference between thermistor and temperature probe is a reflection of heat transfer which can be translated into a measurement of CBF in $\mathrm{ml} / 100 \mathrm{~g} / \mathrm{min}$. A commercial TDF catheter is available, but clinical data using this technology are limited. 


\section{CEREBRAL MICRODIALYSIS}

Cerebral microdialysis (MD) is a well-established laboratory tool that was introduced as a bedside monitor of brain tissue biochemistry more than two decades ago. Because it monitors cellular metabolism as well as substrate supply, MD is able to identify both ischemic and non-ischemic causes of cellular energy dysfunction and the ensuing metabolic crisis [31].

The technical aspects of cerebral MD have been described in detail elsewhere [32]. In brief, a miniature MD catheter is placed into brain tissue and diffusion of molecules across the semi-permeable dialysis membrane at its tip allows collection of substances that pass from the brain extracellular fluid (ECF) into the dialysis fluid. This is collected at regular (usually hourly) intervals and the concentrations of glucose, lactate, pyruvate, glycerol and glutamate can be measured in a semi-automated analyzer at the bedside. Subsequent off-line analysis of the dialysate allows measurement of a myriad of other biomarkers for research purposes.

\section{Interpretation of cerebral microdialysis variables}

Each of the biochemical substances measured in the clinical setting is a marker of a particular cellular process associated with glucose metabolism, hypoxia/ischemia, or cellular energy failure [33]. A dramatic increase in cerebral glucose utilization (cerebral hyperglycolysis) may follow ABI, resulting in critical reductions in cerebral glucose levels despite adequate supply. Glucose is the main substrate for brain metabolism, and periods of low cerebral glucose concentration are associated with unfavourable outcome after TBI [34]. 
Glucose is metabolized via glycolysis to pyruvate which, in the presence of normal oxidative conditions, enters the highly efficient energy-producing tricarboxylic acid (TCA) cycle. Under hypoxic conditions, or if mitochondrial function is compromised, pyruvate is metabolized to lactate outside the TCA cycle, resulting in a lower energy yield. The ECF lactate to pyruvate (LP) ratio is a marker of cellular redox state, and elevated LP ratio is associated with poor outcome. An increased LP ratio may result from both ischemic and non-ischemic causes, and it is therefore important that absolute lactate and pyruvate concentrations are considered when interpreting the LP ratio. An elevated LP ratio in the presence of low pyruvate (and brain tissue oxygen tension) indicates classic ischemia, whereas elevated LP ratio in the presence of normal or high pyruvate indicates a non-ischemic cause, i.e. mitochondrial dysfunction [35]. LP ratio, in combination with ECF glucose levels, therefore provides useful clinical information about the brain's metabolic state, and this ability to assess glucose metabolism is a particular strength of cerebral MD monitoring [36].

Cerebral MD monitored glutamate is a marker of hypoxia/ischemia and excitotoxicity, and glycerol a marker of hypoxia/ischemia-related cell membrane breakdown [33].

Since cerebral MD measures changes at the cellular level, it may identify cerebral compromise before it is detectable clinically or by other monitored variables [37].

\section{Indications for cerebral microdialysis monitoring}


Cerebral MD should be considered in all patients at risk of developing cerebral hypoxia/ischemia, cellular energy failure and glucose deprivation [2]. It has been most widely used in the critical care management of TBI and SAH, but may also have utility after ICH and acute ischemic stroke. Although the timely detection of impending hypoxia/ischemia would be of significant benefit intraoperatively, a recent systematic review reported limited and low quality evidence supporting the use of cerebral MD for diagnostic purposes during neurosurgery [38]. Furthermore, the temporal resolution of the only commercially available clinical system (hourly sampling rate) is unlikely to be adequate for intraoperative monitoring. A continuous rapid-sampling cerebral MD technique has been described for research use, but such systems are not currently available for clinical applications.

The heterogeneity of the pathophysiological changes after ABI means that brain chemistry varies in different regions of the brain. Cerebral MD is a focal technique so changes in tissue chemistry must be interpreted with knowledge of catheter location. This can be confirmed by CT visualization of a gold marker at the MD catheter tip. Placement of the catheter in 'at risk' tissue is generally advocated, to facilitate assessment of biochemical changes in the region most susceptible to secondary injury [39].

\section{Reference values and thresholds for intervention}

Absolute normal or abnormal thresholds for monitored brain tissue chemistry are difficult to define based on current data, and a combination of variables has most often been used to relate brain chemistry to outcome. The importance of distinguishing between normal values derived from studies in awake and anesthetised 
patients undergoing surgery for benign intracranial lesions from those that characterize pathophysiological disturbance of brain chemistry has recently been emphasized [39]. Further, the trend of variables is as important, or possibly more important, than individual measurements or threshold values.

Cerebral MD monitored glucose, lactate and LP ratio are now considered more useful in the clinical management of brain injured patients than glutamate and glycerol [39]. Values that are usually recommended to guide clinical intervention are glucose $<0.8$ $\mathrm{mmol} / \mathrm{L}$ and LP ratio $>40$ [34], although a lower LP ratio threshold is recommended by some [33]. When interpreting an elevated LP ratio, lactate concentration $>4$ $\mathrm{mmol} / \mathrm{l}$ is generally considered abnormal [34].

Cerebral hypoglycemia in association with elevated LP ratio is a sign of severe hypoxia/ischemia. If brain glucose is very low $(<0.2 \mathrm{mmol} / \mathrm{l})$, a trial of increasing serum glucose (even if within normal limits) should be considered [39]. If the LP ratio indicates ischemia, augmentation of CPP is a therapeutic option, whereas if elevated LP ratio is associated with low brain tissue oxygenation, several interventions that improve oxygen delivery, including judicious increases in $\mathrm{CPP}$ or $\mathrm{FiO}_{2}$ or correction of anemia, can be considered. Although the LP ratio has been used to guide CPP optimization after TBI, some studies have found that it may be abnormal despite CPP values that are customarily considered to be adequate [40]. This is perhaps unsurprising given the several non-ischemic causes of elevated LP ratio, and further highlights the importance of using multi-modality physiological data to guide individualized patient management. 


\section{Future perspectives}

Cerebral MD has contributed substantially to our understanding of the pathophysiology of brain injury, but its clinical utility is still debated. While there is a large body of evidence demonstrating an association between abnormal brain tissue chemistry and clinical outcome after ABI, there are no data to confirm whether cerebral MD-guided therapy can influence outcome. Future clinical research should focus on assessing the clinical effectiveness of cerebral MD as a component of multimodality monitoring to guide decision-making in acute brain injured patients, and its integration into treatment paradigms in neurocritical care [39].

\section{NEUROINFORMATICS}

Multimodality neuromonitoring produces large and complex datasets and, to maximize the clinical effectiveness of monitoring, systems have been developed to analyze and present clinically relevant data in a user-friendly and timely manner at the bedside [2]. Some systems are commercially available, although many have been designed around the needs of individual researchers or institutions [41]. There are several challenges that hinder the integration of data from multiple monitoring modalities, including situations in which one or more monitored variables remain normal in the face of derangements in others, and lack of standardization across different monitoring devices which have often been developed as standalone tools. In the future, incorporation of advanced algorithms is likely to allow automatic recognition and rejection of anticipated and expected fluctuations in data, such as transient increases in ICP associated with suctioning or repositioning of a patient. 
There is also interest in the incorporation of computational models of cerebral oxygenation, hemodynamics and metabolism to interpret complex datasets and provide timely summary outputs that can guide clinical decision making [42]. Such approaches can also be used to produce patient-specific simulations of clinically important but unmeasured physiological variables such as cerebral metabolism. Model-based interpretation of multimodal neuromontoring data has potential to provide clinicians with information about the underlying processes that are driving the (patho) physiological state of the brain, rather than simply the endpoints of the injurious processes.

\section{CONCLUSIONS}

There is now clear evidence that no single neuromonitor can comprehensively detect all instances of cerebral compromise, and this has driven the development of multimodality neuromonitoring in neurocritical care. The continuous monitoring of multiple physiological variables, including ICP, CPP, cerebral oxygenation, brain chemistry and electrophysiology, allows individualized, targeted treatment guided by actual physiological derangements rather than by generic and often arbitrarily defined thresholds. A multimodal monitoring approach also permits cross-validation between monitored variables and improves confidence in treatment decision making. However, it remains unclear how a derangement in one physiological variable in the presence of normal values in others should be managed. Crucial to the widespread implementation of multimodality neuromonitoring is the development of high-quality outcomes studies to determine whether adoption of a multimodal neuromonitoring approach improves outcomes, and which modalities are more useful than others in guiding treatment of the acutely injured brain. 


\section{REFERENCES}

1. Smith M (2008) Monitoring intracranial pressure in traumatic brain injury. Anesth Analg 106:240-248. doi: 10.1213/01.ane.0000297296.52006.8e

2. Le Roux P, Menon DK, Citerio G, et al. (2014) Consensus summary statement of the International Multidisciplinary Consensus Conference on Multimodality Monitoring in Neurocritical Care : a statement for healthcare professionals from the Neurocritical Care Society and the European Society of Intensive Care Medicine. Intensive Care Med 40:1189-1209. doi: 10.1007/s00134-0143369-6

3. Kristiansson H, Nissborg E, Bartek J, et al. (2013) Measuring elevated intracranial pressure through noninvasive methods: a review of the literature. J Neurosurg Anesthesiol 25:372-385. doi: 10.1097/ANA.0b013e31829795ce

4. Oddo M, Villa F, Citerio G (2012) Brain multimodality monitoring: an update. Curr Opin Crit Care 18:111-118. doi: 10.1097/MCC.0b013e32835132a5

5. Brain Trauma Foundation, (2007) Guidelines for the management of severe traumatic brain injury. J Neurotrauma 24 Suppl 1:S1-106. doi: 10.1089/neu.2007.9999

6. Stocchetti N, Picetti E, Berardino M, et al. (2014) Clinical applications of intracranial pressure monitoring in traumatic brain injury : report of the Milan consensus conference. Acta Neurochir (Wien) 156:1615-1622. doi: $10.1007 / \mathrm{s} 00701-014-2127-4$

7. Sandsmark DK, Kumar MA, Park S, Levine JM (2012) Multimodal monitoring in subarachnoid hemorrhage. Stroke 43:1440-1445. doi: 10.1161/STROKEAHA.111.639906

8. Kirkman MA, Smith M (2013) Supratentorial intracerebral hemorrhage: a review of the underlying pathophysiology and its relevance for multimodality neuromonitoring in neurointensive care. J Neurosurg Anesthesiol 25:228-239. doi: 10.1097/ANA.0b013e3182836059

9. Asgari S, Bergsneider M, Hamilton R, et al. (2011) Consistent changes in intracranial pressure waveform morphology induced by acute hypercapnic cerebral vasodilatation. Neurocrit Care 15:55-62. doi: 10.1007/s12028-0109463-x

10. Yuan Q, Wu X, Sun Y, et al. (2015) Impact of intracranial pressure monitoring on mortality in patients with traumatic brain injury: a systematic review and meta-analysis. J Neurosurg 122:574-587. doi: 10.3171/2014.10.JNS1460

11. Chesnut RM, Temkin N, Carney N, et al. (2012) A trial of intracranial-pressure monitoring in traumatic brain injury. N Engl J Med 367:2471-2481. doi:

10.1056/NEJMoa1207363 
12. Cremer OL, van Dijk GW, van Wensen E, et al. (2005) Effect of intracranial pressure monitoring and targeted intensive care on functional outcome after severe head injury. Crit Care Med 33:2207-2213.

13. Oddo M, Levine JM, Mackenzie L, et al. (2011) Brain hypoxia is associated with short-term outcome after severe traumatic brain injury independently of intracranial hypertension and low cerebral perfusion pressure. Neurosurgery 69:1037-1045. doi: 10.1227/NEU.0b013e3182287ca7

14. Bouzat P, Marques-Vidal P, Zerlauth J-B, et al. (2015) Accuracy of brain multimodal monitoring to detect cerebral hypoperfusion after traumatic brain injury. Crit Care Med 43:445-452. doi: 10.1097/CCM.0000000000000720

15. Kirkman MA, Smith M (2014) Intracranial pressure monitoring, cerebral perfusion pressure estimation, and ICP/CPP-guided therapy: a standard of care or optional extra after brain injury? Br J Anaesth 112:35-46. doi: $10.1093 / \mathrm{bja} /$ aet 418

16. Kosty JA, Leroux PD, Levine J, et al. (2013) Brief report: a comparison of clinical and research practices in measuring cerebral perfusion pressure: a literature review and practitioner survey. Anesth Analg 117:694-698. doi: 10.1213/ANE.0b013e31829cc765

17. Smith M (2015) Cerebral perfusion pressure. British Journal of Anaesthesia 115:488-490. doi: 10.1093/bja/aev230

18. Czosnyka M, Pickard JD (2004) Monitoring and interpretation of intracranial pressure. J Neurol Neurosurg Psychiatry 75:813-821.

19. Aries MJH, Czosnyka M, Budohoski KP, et al. (2012) Continuous determination of optimal cerebral perfusion pressure in traumatic brain injury. Crit Care Med 40:2456-2463. doi: 10.1097/CCM.0b013e3182514eb6

20. Ono M, Zheng Y, Joshi B, et al. (2013) Validation of a stand-alone nearinfrared spectroscopy system for monitoring cerebral autoregulation during cardiac surgery. Anesth Analg 116:198-204. doi:

10.1213/ANE.0b013e318271fb10

21. Hori D, Hogue CW, Shah A, et al. (2015) Cerebral Autoregulation Monitoring with Ultrasound-Tagged Near-Infrared Spectroscopy in Cardiac Surgery Patients. Anesth Analg 121:1187-1193. doi: 10.1213/ANE.0000000000000930

22. Lazaridis C, Andrews CM (2014) Brain Tissue Oxygenation, Lactate-Pyruvate Ratio, and Cerebrovascular Pressure Reactivity Monitoring in Severe Traumatic Brain Injury: Systematic Review and Viewpoint. Neurocrit Care 21:345-355. doi: 10.1007/s12028-014-0007-7

23. Diedler J, Karpel-Massler G, Sykora M, et al. (2010) Autoregulation and brain metabolism in the perihematomal region of spontaneous intracerebral hemorrhage: an observational pilot study. J Neurol Sci 295:16-22. doi: 10.1016/j.jns.2010.05.027 
24. Ono M, Brady K, Easley RB, et al. (2014) Duration and magnitude of blood pressure below cerebral autoregulation threshold during cardiopulmonary bypass is associated with major morbidity and operative mortality. $\mathrm{J}$ Thorac Cardiovasc Surg 147:483-489. doi: 10.1016/j.jtcvs.2013.07.069

25. Moritz S, Kasprzak P, Arlt M, et al. (2007) Accuracy of cerebral monitoring in detecting cerebral ischemia during carotid endarterectomy: a comparison of transcranial Doppler sonography, near-infrared spectroscopy, stump pressure, and somatosensory evoked potentials. Anesthesiology 107:563-569. doi: 10.1097/01.anes.0000281894.69422.ff

26. Ringelstein EB, Droste DW, Babikian VL, et al. (1998) Consensus on microembolus detection by TCD. International Consensus Group on Microembolus Detection. Stroke 29:725-729.

27. Mascia L, Fedorko L, terBrugge K, et al. (2003) The accuracy of transcranial Doppler to detect vasospasm in patients with aneurysmal subarachnoid hemorrhage. Intensive Care Med 29:1088-1094. doi: 10.1007/s00134-003$1780-5$

28. Lindegaard KF, Nornes H, Bakke SJ, et al. (1989) Cerebral vasospasm diagnosis by means of angiography and blood velocity measurements. Acta Neurochir (Wien) 100:12-24.

29. Fontanella M, Valfrè W, Benech F, et al. (2008) Vasospasm after SAH due to aneurysm rupture of the anterior circle of Willis: value of TCD monitoring. Neurol Res 30:256-261. doi: 10.1179/016164107X229939

30. Carrera E, Schmidt JM, Oddo M, et al. (2009) Transcranial Doppler for predicting delayed cerebral ischemia after subarachnoid hemorrhage. Neurosurgery 65:316-23- discussion 323-4. doi: 10.1227/01.NEU.0000349209.69973.88

31. Vespa P, Bergsneider M, Hattori N, et al. (2005) Metabolic crisis without brain ischemia is common after traumatic brain injury: a combined microdialysis and positron emission tomography study. J Cereb Blood Flow Metab 25:763-774. doi: $10.1038 /$ sj.jcbfm.9600073

32. de Lima Oliveira M, Kairalla AC, Fonoff ET, et al. (2014) Cerebral microdialysis in traumatic brain injury and subarachnoid hemorrhage: state of the art. Neurocrit Care 21:152-162. doi: 10.1007/s12028-013-9884-4

33. Tisdall MM, Smith M (2006) Cerebral microdialysis: research technique or clinical tool. Br J Anaesth 97:18-25. doi: 10.1093/bja/ael109

34. Timofeev I, Carpenter KLH, Nortje J, et al. (2011) Cerebral extracellular chemistry and outcome following traumatic brain injury: a microdialysis study of 223 patients. Brain 134:484-494. doi: 10.1093/brain/awq353

35. Larach DB, Kofke WA, Le Roux P (2011) Potential Non-Hypoxic/Ischemic Causes of Increased Cerebral Interstitial Fluid Lactate/Pyruvate Ratio: A 
Review of Available Literature. Neurocrit Care 15:609-622.

36. Jalloh I, Carpenter KLH, Helmy A, et al. (2015) Glucose metabolism following human traumatic brain injury: methods of assessment and pathophysiological findings. Metab Brain Dis 30:615-632. doi: 10.1007/s11011-014-9628-y

37. Belli A, Sen J, Petzold A, et al. (2008) Metabolic failure precedes intracranial pressure rises in traumatic brain injury: a microdialysis study. Acta Neurochir (Wien) 150:461-9- discussion 470. doi: 10.1007/s00701-008-1580-3

38. Bossers SM, de Boer RDH, Boer C, Peerdeman SM (2013) The diagnostic accuracy of brain microdialysis during surgery: a qualitative systematic review. Acta Neurochir (Wien) 155:345-353. doi: 10.1007/s00701-012-1582-Z

39. Hutchinson PJ, Jalloh I, Helmy A, et al. (2015) Consensus statement from the 2014 International Microdialysis Forum. Intensive Care Med 41:1517-1528. doi: 10.1007/s00134-015-3930-y

40. Vespa PM, O'Phelan K, McArthur D, et al. (2007) Pericontusional brain tissue exhibits persistent elevation of lactate/pyruvate ratio independent of cerebral perfusion pressure. Critical Care Medicine 35:1153-1160. doi: 10.1097/01.CCM.0000259466.66310.4F

41. Sorani MD, Hemphill JC, Morabito D, et al. (2007) New approaches to physiological informatics in neurocritical care. Neurocrit Care 7:45-52. doi: $10.1007 / \mathrm{s} 12028-007-0043-7$

42. Caldwell M, Hapuarachchi T, Highton D, et al. (2015) BrainSignals Revisited: Simplifying a Computational Model of Cerebral Physiology. PLoS ONE 10:e0126695. doi: 10.1371/journal.pone.0126695 


\section{Table 1}

\section{Indications for neuromonitoring}

- Monitoring the healthy but 'at risk' brain

- intraoperative monitoring during selected procedures including cardiac and carotid surgery

- Early detection of secondary adverse events after acute brain injury

- intracranial hypertension

- reduced cerebral perfusion

- impaired cerebral glucose delivery/utilization

- cerebral hypoxia/ischemia

- cellular energy failure

- non-convulsive seizures

- Guiding individualized, patient-specific therapy after acute brain injury

- optimization of intracranial and cerebral perfusion pressures

- optimization of brain tissue oxygenation

- optimization of cerebral glucose delivery/utilization

- monitoring cerebral vasospasm after subarachnoid hemorrhage

- prognostication 


\section{Table 2}

Indications for intracranial pressure monitoring in traumatic brain injury

\section{Brain Trauma Foundation 2007 [5]}

- all salvageable patients with severe TBI and an abnormal cranial CT scan

- a normal scan and two or more of
$\circ$ age $>40$ years
$\circ$ unilateral or bilateral motor posturing
○ systolic blood pressure $<90 \mathrm{mmHg}$

\section{The Milan consensus conference 2014 [6]}

- ICP should be monitored

- in comatose patients with:

- cerebral contusions

- when clinical examination is unreliable

- interruption of sedation to check neurologic status is dangerous

- following secondary decompressive craniectomy

- ICP monitoring should be considered

- after evacuation of an acute supratentorial intracranial hematoma in salvageable patients at increased risk of intracranial hypertension, including those with:

- GCS motor score $\leq 5$

- pupillary abnormalities

- prolonged/severe hypoxia and/or hypotension

- cranial CT findings suggestive of raised ICP

- intra-operative brain swelling

- interruption of sedation to check neurologic status is dangerous

- in patients with extracranial injuries requiring multiple surgical procedures and/or prolonged analgesia and sedation 